\title{
DESIGN AND SIMULATION OF A HIGH EFFICIENCY ROTMAN LENS FOR MM-WAVE SENSING APPLICATIONS
}

\author{
L. T. HALL, D. ABBOTT \\ Centre for Biomedical Engineering \\ Department of Electrical and Electronic Engineering \\ University of Adelaide SA 5005 \\ E-mail: 1thall@eleceng.adelaide.edu.au
}

H. J. HANSEN

DSTO

EWD, PO Box 1500, Salisbury SA 5108

E-mail: hedley.hansen@dsto.defence.gov.au

\begin{abstract}
Multi-beam approaches using beam-forming antenna array architectures have been identified as one solution for overcoming the limited fields-of-view provided by highly directional mm-wave sensors. Rotman lenses offer a compact, rugged and reliable alternative to electronically scanned antenna technologies but architectures that operate at frequencies $<20 \mathrm{GHz}$ perform poorly at higher frequencies on account of greater losses and dispersion. This paper outlines the design process for providing Rotman-based lenses, examining various levels of simulation that are needed for designs that function at $\mathrm{K}$ and $\mathrm{W}$-band frequencies. The impact of using mictrostrip structures is demonstrated.
\end{abstract}

\section{Introduction}

Sensors operating in the mm-wave $(20-100 \mathrm{GHz})$ band have better poor weather performance than those operating at IR and visible wavelengths and provide better spatial resolution (and target discrimination) than microwave systems.

Beamforming mm-wave antennas have numerous applications including radar, communication systems, synthetic vision schemes and automobile collision-avoidance systems. The strategy is to implement a beamforming Rotman lens-based antenna array [1], operating at $35 \mathrm{GHz}$ that can be monolithically fabricated so that it provides a low-cost testing platform of the design for a further version at the higher $94 \mathrm{GHz}$. These frequencies are chosen because they are centres of atmospheric transmission bands, with the presence of $\mathrm{O}_{2}$ and $\mathrm{H}_{2} \mathrm{O}$ in the atmosphere causing absorption of mmwaves at 60 and $22 \mathrm{GHz}$ respectively. Naturally occurring 35 and $94 \mathrm{GHz}$ radiation propagates through cloud, smoke, fog and other such aerosols, as seen by the attenuation curve in Fig. 1. This property makes the use of these frequencies extremely attractive for remote sensing applications.

\section{Specifications of Integrated Design}

The initial design that is being considered is a $35 \mathrm{GHz}$ lens for steering seven beams with a $20^{\circ}$ scan angle. This provides a simple prototype to enable rapid development of the skills required for the design's manufacture at affordable cost. In addition in this design, the half-wavelength separation of beamports is achievable and therefore spillover losses can be kept low.

The lens and the feeds are to be implemented using microstrip fed by dipolar antenna elements. This architecture is best suited for providing a compact structure that is planar and is compatible with printed and monolithic fabrication. It is estimated that a fifteen element array aperture of $50 \mathrm{~mm}$ is achievable at $37 \mathrm{GHz}$ and an aperture of $22 \mathrm{~mm}$ at $94 \mathrm{GHz}$. Apertures of these sizes are suitable for collision-avoidance applications. The second implementation of the design will utilise a horn-fed array since such a lens, as an aperture feed, promises high gain multi-beam coverage of a $20^{\circ}$ field-of-view. 
This is desired in electronic warfare applications. Aspects of the first implementation are addressed in this paper.

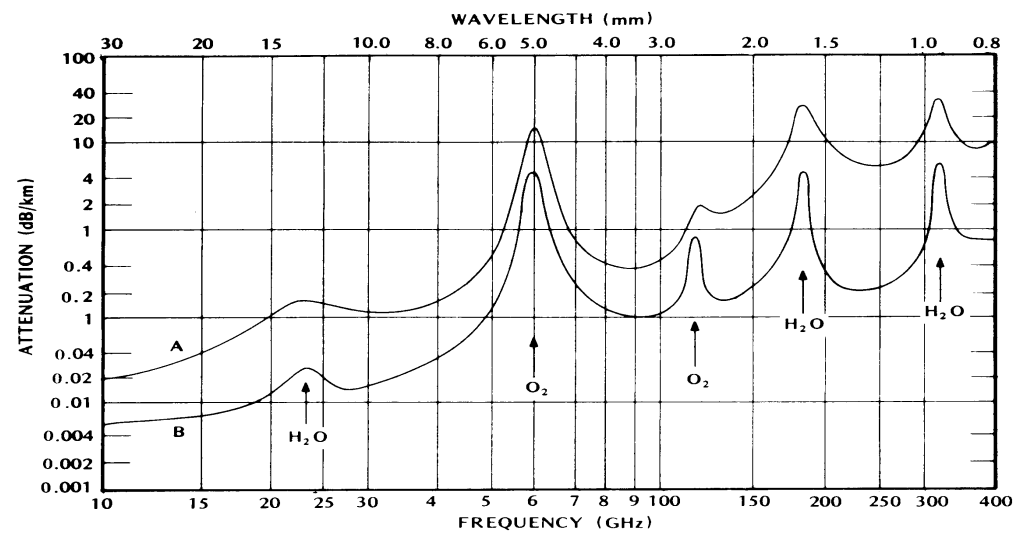

Figure 1. The one way attenuation of electromagnetic radiation in the troposphere [2].

\section{Simulation}

A Rotman lens consists of a parallel plate region with beam ports and array ports distributed along opposite contours. The central beamport provides equal path lengths to each array element. An offset beam produces a path length and hence a phase gradient along an array giving a steered beam.

In order to design a lens and ensure that it will perform at mm-wave wave frequencies, three different levels of simulation are being considered. Some results associated with the first couple of these levels are reported here.

Level one simulation involves the numerical optimisation of the Rotman geometry using ideal models [3-4]. The lens is modelled using geometric optics. No coupling between input and output ports is considered with all ports matched and reflections and attenuation ignored.

Level two simulation addresses the non-ideal effects of the microstrip and how adopting various antenna element options affect performance [5]. Level two simulations include optimising the bandwidth performance of the lens.

Level three simulation utilises an electromagnetic simulation program such as APLAC to calculate the S-matrix of various sections of the design such as ports and junctions. At this level, effects considered include the coupling between adjacent and other input and output ports, reflections, and unmatched ports. An electromagnetic simulation of the antenna element feeds could also be included. It is expected that only through the careful implementation of level three studies that our model would be able to accurately predict the performance of the lens structure.

\section{Results}

\section{4-1 Level 1 Ideal Modelling}

Scattering matrices have been used exclusively throughout the simulation because the matrix approach allows a complete model of the lens to be developed [6]. Initially three scattering matrices are generated, $S_{B}$ accounting for the Rotman lens body, $S_{W}$ for the path delays between antenna elements and associated antenna ports on the inner lens contour and $S_{A}$, for the phase delays between incoming rays onto the antenna array. Combining these matrices provides the output at each port due to a given 
incident wave.

The design of the lens or matrix $S_{a}$ is governed by the Rotman-Turner design equations that are derived by viewing the geometry of the lens. Fig. 2 shows the layout of the fifteen antenna element-, seven beam port configuration under consideration where the opposite contours are clearly shown. A normalised focal length ratio of $g=1.01$ is used with the off-axis focal point angle $\alpha=10^{\circ}$. Fig. 3

shows the desired path lengths that are needed to link antenna element to corresponding antenna port. Fig. 4 displays the associated maximum phase error with angle. For the three focal points, there is no phase error at all and between these points the phase error remains under $0.2^{\circ}$. Fig. .5 then displays the idealised seven-beam radiation pattern. The average side lobe level for an array aperture with antenna elements spaced $0.8 \lambda$ apart is $-30 \mathrm{~dB}$. Clearly, the amplitude and phase performance of the design is acceptable.

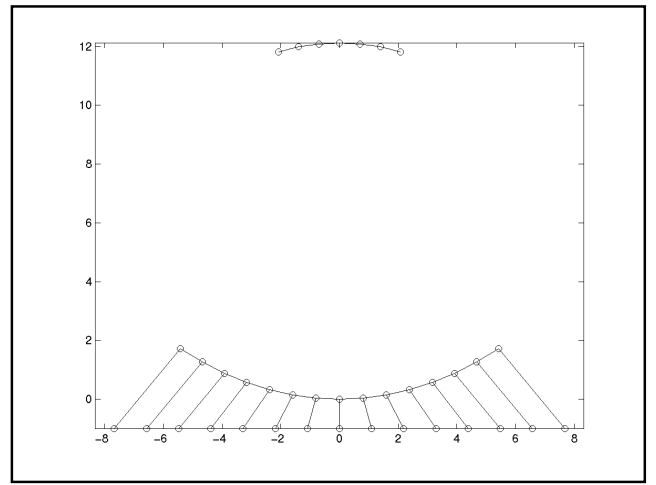

Figure 2. The lens layout.

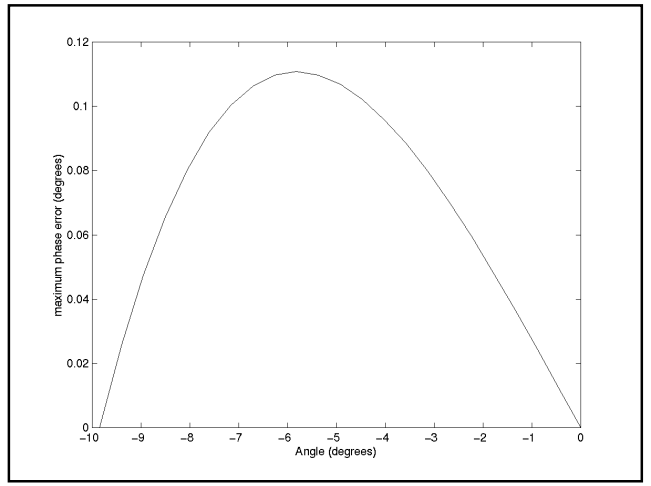

Figure 4. Phase error versus beam angle.

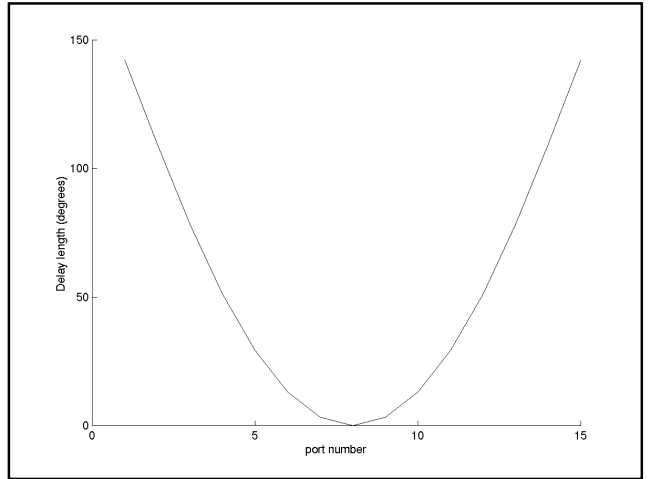

Figure 3. Path lengths associated with antenna elements.

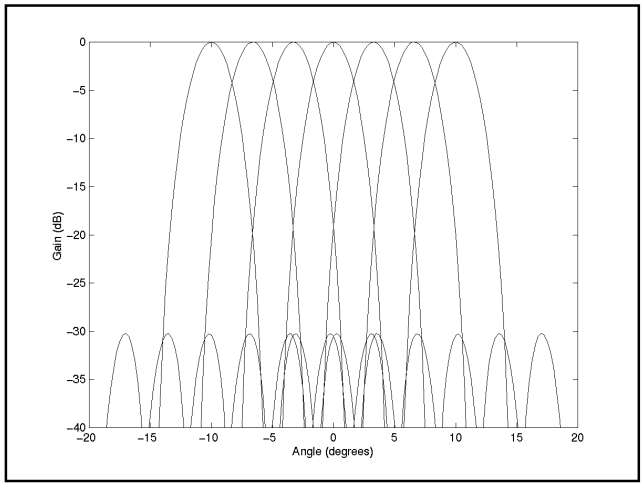

Figure 5. The seven-beam radiation pattern.

\subsection{Level 2 - Non-Ideal Modelling Used}

We now show the adverse effect on performance of using non-ideal antenna elements within a microstrip lens structure. For this simulation, the $S_{A}$ matrix is altered to account for attenuation in microstrip and an $S_{W}$ matrix is considered that uses non-ideal dipoles as antenna elements.

Fig. 6 shows that attenuation in microstrip will account for $<2 \mathrm{~dB}$ drop in amplitude of the main beams across the $30^{\circ}$ range of scan angles. Fig. 7 displays a curving of the response due to the gain associated with the array aperture. The amplitudes of the main beams decrease by $-0.5 \mathrm{~dB}$ towards the intended maximum angle of $\pm 10^{\circ}$. 


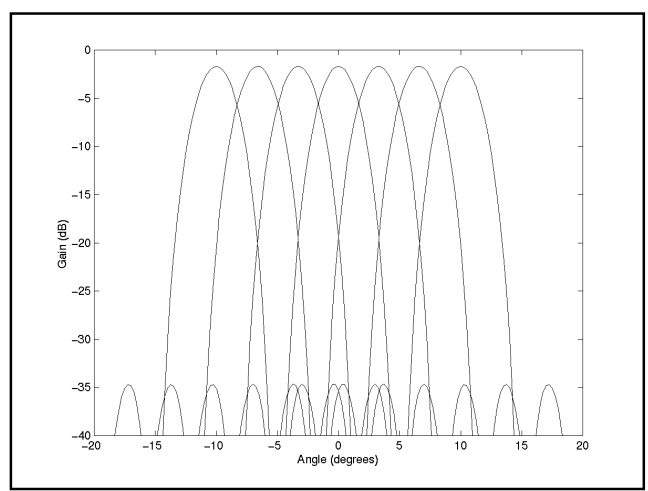

Figure. 6. Gain with microstrip attenuation.

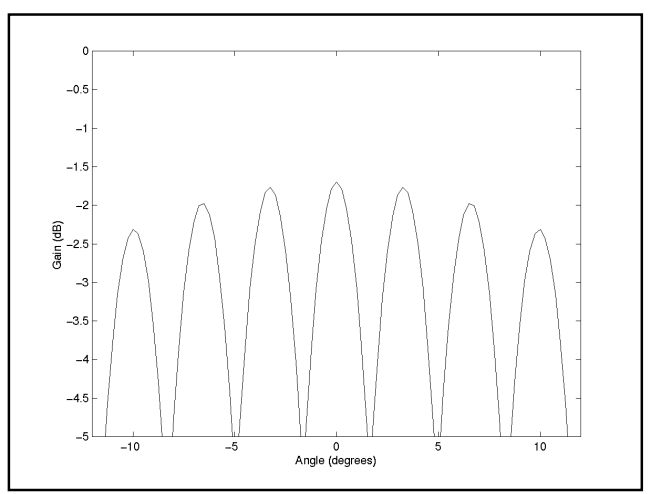

Figure 7. Gain with attenuation and antenna.

\section{Discussion}

The modelling shows that a working integrated planar lens array, for mm-wave operation in short range collision-avoidance type applications, is achievable using microstrip technology. Our calculations show that adopting a microstrip based design should be responsible for an attenuation in mainbeam loss $\sim 2 \mathrm{~dB}$. Previous studies of similar designs have reported $2.5 \mathrm{~dB}$ mainbeam losses overall [7] so it is clear that minimising the microstrip paths within the lens will be a major factor in providing an optimally performing structure.

The results reported are preliminary because our model does not include any of the electromagnetic effects such as coupling between ports, reflections and the existence of propagating multiple modes. We intend to use electromagnetic simulation programs to generate the scattering matrices of geometry's which include those associated with the interfacing structures into and out of the body of the Rotman lens. Ultimately, our approach should predict the sidelobe levels and mainbeam gains that are to be achieved with a fabricated device.

\section{Acknowledgements}

This research is supported by funding from the ARC, the DSTO RF Hub, and the Sir Ross \& Sir Keith Smith Fund.

Thanks to Abdulla Mohamed, Andrew Campbell, and David Goodfellow for their work and help on Rotman lens design.

\section{References}

[1] W. Rotman, "Wide angle microwave lens for line source applications", IEEE Trans. Antennas Propagate. AP-11, pp 623-632, Nov 1963.

[2] L. A. Klein, "Millimeter-Wave and Infra-Red Multisensor Design and Signal Processing", Artech House, pp 58, 1997.

[3] R. C. Hansen, "Design Trades For Rotman Lenses", IEEE Trans. Antennas Propagate, Vol 39, No 4, pp 464-472, Nov 1991.

[4] A. F. Peterson, "Scattering Matrix Integral Equation Analysis for the Design of a Waveguide Rotman Lens", IEEE Trans. Antennas Propagate, Vol 47, No 5, pp 870-878, May 1999.

[5] D. M. Pozar, Microwave Engineering, New York: Wiley, 1998.

[6] A. F. Peterson, Computational Methods for Electromagnetics, New York: IEEE Press, 1998.

[7] D. Abbott and A.J. Parfitt, Collision avoidance device using passive mm-wave array based insect vision, Proc. MICRO 97 Melbourne, pp201-203, Oct 1997 